\title{
Noninvasive Cardiac Testing-A New Routine in Evaluation of Renal Transplant Candidates
}

\author{
Marina Moguilevitch, Ellise Delphin \\ Montefiore Medical Center, Albert Einstein College of Medicine, New York, USA \\ Email: mmoguile@montefiore.org
}

Received 15 February 2014; revised 14 March 2014; accepted 8 April 2014

Copyright (C) 2014 by authors and Scientific Research Publishing Inc.

This work is licensed under the Creative Commons Attribution International License (CC BY). http://creativecommons.org/licenses/by/4.0/

(c) (i) Open Access

\begin{abstract}
We report a case of a young kidney transplant candidate presented with severe and clinically completely asymptomatic mitral stenosis. The bedside Echocardiogram was helpful to establish the diagnosis. The surgery had to be postponed and the patient was referred to a cardiologist for the further workup. This case report provides solid evidence that the renal transplant candidates are a very unique group of patients with specific disease development course. Their preoperative workup cannot be based on widely used guidelines from American College of Cardiology and American Heart Association that are relying on clinical symptoms and presentation of cardiac disease. All physicians who are involved in the care of these patients and making decisions about their candidacy for the transplantation should take into account severity and very often asymptomatic presentation of the cardiovascular complications of the end stage renal disease. This case provides a good example of the importance of the multispecialty involvement and coordination in perioperative care for prospective kidney transplant candidates.
\end{abstract}

\section{Keywords}

Kidney Transplant, Cardiac Workup, Critical Mitral Stenosis

\section{Introduction}

Renal transplantation became a preferred treatment option for most patients with end-stage renal disease (ESRD). The first year graft survival ranges between 90\% and 95\% [1]. With this high success rate and increasing demand, the transplant list waiting time has been extended significantly. According to the Organ Procurement and Transplant Network (OPTN) records, nearly 85,000 candidates were on the waiting list for kidney 
transplantation in 2010 and only 17,700 kidney transplant were performed [2]. All these factors determine the development of a new trend to accept older patients, patients with obesity and cardiovascular disease for the kidney transplantation. Cardiovascular disease-including congestive heart failure (CHF), coronary artery disease (CAD), cerebrovascular disease, and peripheral vascular disease (PVD) - common in patients with ESRD, and the risk of cardiac death in dialysis patients has been shown to be $10-20$ times greater than that in general patient population [3].

That is why conducting clinically appropriate and cost-effective cardiac evaluation among transplant candidates presents a challenging task for the clinicians.

\section{Case Presentation}

The patient is a 28-year-old male with past medical history of hypertension (HTN) secondary to focal glomerulosclerosis, end stage renal disease on hemodialysis for the past 16 years. He demonstrated good exercise tolerance by walking more than 15 blocks a day and lifting weights for more than an hour few times per week without exhibiting any symptoms. The patient was scheduled to undergo kidney transplantation. As a part of a routine work up he presented for a preoperative cardiac evaluation. His physical examination was remarkable for 3/6 holosystolic murmur (HSM) at the left lateral sternal boarder and increased left ventricular impulse. Taking in consideration the results of his physical examination the patient was referred for an echocardiography (Echo).

His laboratory studies revealed hemoglobin $11.4 \mathrm{~g} / \mathrm{dl}$, platelets 176,000, potassium $5.8 \mathrm{meq} / \mathrm{l}$, creatinine 16 $\mathrm{mg} / \mathrm{dl}$, BUN $48 \mathrm{mg} / \mathrm{dl}$, calculated GFR $6 \mathrm{ml} / \mathrm{min}$.

The patient missed his scheduled Echo appointment. The preoperative Echo study was performed on the day when the patient was called as soon as a suitable organ became available. His echocardiogram demonstrated severe concentric left ventricular (LV) remodeling with left ventricular hyperkinesis and estimated EF $85 \%$. The exam also revealed severe mitral valve stenosis with pressure gradient of $16.9 \mathrm{mmHg}$. The right and left ventricles appeared to be normal with minimal tricuspid valve regurgitation.

The transplant surgery was postponed and the patient was referred to a cardiologist for the further work up and possible mitral valve replacement.

\section{Discussion}

The development of kidney failure associated with annual mortality approaching 25 percent of affected patients population. Cardiovascular events are the leading cause of mortality in ESRD patients, accounting for over 50 percent of all deaths [4]. Kidney transplantation is considered the standard of care for this high-risk patients largely because of up to 10 -fold reduction rate of cardiovascular events compared to the dialysis population [3].

The pathogenesis of cardiac disease development is unique in this patient population. In $27 \%$ of hemodialysis patients CAD is caused by non-atherosclerotic disease [5]. Left ventricular (LV) structure and function are altered as a result of a chronic fluid overload. It causes LV hypertrophy with increased myocyte length and thickening. Initially, the LV hypertrophy is adaptive and beneficial in maintaining stable wall stress. However, continuing LV overload leads to myocyte death, development of myocardial fibrosis, and diastolic dysfunction. As the result of these changes the LV volume-pressure curve is displaced to the left. At this stage the small changes in LV volume result in large changes in LV pressure, predisposing to symptomatic LV failure. Myocyte death induces LV dilation and eventually leads to systolic dysfunction. All above mentioned processes as well as hyperparathyroidism, and uremia compromise coronary vessels perfusion. Blood flow overload causes not only LV remodeling but also vascular remodeling promoting the development of accelerated arteriosclerosis with thickened, dilated, and non-compliant arteries.

An observational study in the ESRD population has demonstrated an increased incidence of aortic and mitral valve calcification mostly related to calcium and phosphate metabolism [6]. The same study emphasized an asymptomatic development and a rapid rate of progression of valvular disease in renal failure patients. Emerging research in the dialysis population has suggested that other factors such as neopterin (a marker of cellular immune system activation) may play a roll in the rapid progression of valvular disease [7]. In case of the mitral valve disease development valvular calcification may begin as a mitral annular calcification with encroachment into both mitral leaflets leading to mitral regurgitation (MR), mitral stenosis (MS) and both. In the clinical assessment of the severity of the mitral valve disease especially in the case of regurgitation it is very important to evaluate the patient immediately after dialysis. The volume status of the patient will significantly affect the de- 
gree of MR.

All these unique clinical characteristics and atypical presentation of the cardiac disease in the ESRD patient population made the existing American College of Cardiology (ACC) and American Heart Association (AHA) "Guidelines on Perioperative Cardiovascular Evaluation and Care for Non-Cardiac Surgery" not applicable for this group of patients. It is extremely important to remember that "absence of cardiac symptoms" may differ in transplant candidates compared with the general population. The ACC/AHA guidelines were created in consideration that non-cardiac surgery will be performed shortly after evaluation and any management decisions will affect perioperative short-term outcome. In contrast, cardiac evaluation and interventions in transplant candidates should be considered from both short and long term prospective.

The American Heart Association and American College of Cardiology Foundation (AHA/ACCF) conducted a comprehensive review of the literature relevant to the perioperative cardiac evaluation of potential kidney and liver transplant recipients. This review included an incidence and prevalence of CAD and valvular disease in this patient population; incidence of major adverse cardiac events before and after transplantation; accuracy of clinical risk markers, symptoms, and non-invasive testing before and after transplant listing, as well as clinical outcomes of revascularization and the medical management of atherosclerosis [8]. The most compelling goals of this review are the perioperative cardiac risk evaluation and reduction of the morbidity and mortality in transplant candidates.

According to the AHA/ACCF scientific statement, it is reasonable to perform preoperative assessment of left ventricular function by echocardiography (Class IIa evidence). Overall, the management of valvular heart disease in renal transplant candidates is similar to that in the general population [9].

However, there are few specific issues which are typical for renal transplant candidates only. First, the large retrospective study of 35,215 patients on the kidney transplantation waitlist suggested that uncorrected valve disease is a barrier to transplantation [10]. Also, transplantation rates were not reduced among the patients with surgically corrected valvular disease. Hospitalization rates for the heart failure were lower after transplantation. Second, ESRD patients have increased incidence of the aortic and mitral valve disease that requires valve replacement surgery [11].

Unfortunately, in spite of improved renal function after transplantation nearly $40 \%$ of the patients have experienced a cardiovascular event at 36 months after the surgery with congestive heart failure and myocardial infarction being the most common events [12].

\section{Conclusion}

Taking into consideration all available scientific evidence and the history of our patient presentation, we can conclude that preoperative non-invasive cardiac testing of transplant candidates might yield findings that can affect the decision of the particular patient's transplant candidacy and warrant interventions before listing.

\section{References}

[1] Port, F.K., Merion, R.M., Finley, M.P., et al. (2007) Trends in Organ Donation and Transplantation in the United States, 1996-2005. American Journal of Transplantation, 7, 1319-1326. http://dx.doi.org/10.1111/j.1600-6143.2007.01778.x

[2] Health Resources and Services Administration (2011) Organ Procurement and Transplant Network Database. http://optn.transplant.hrsa.gov/

[3] Foley, R.N., Parfrey, P.S. and Sarnak, M.J. (1998) Clinical Epidemiology of Cardiovascular Disease in Chronic Renal Disease. American Journal of Kidney Disease, 32, S112. http://dx.doi.org/10.1053/ajkd.1998.v32.pm9820470

[4] Sato, K., Tadokoro, F., Ishida, K., Matsuzawa, K. and Nakayama, Y. (1994) Causes of Death after Renal Transplantation. A Long Term Follow-Up Study. Transplant Proceedings, 26, 2017-2018.

[5] Rostand, R.G., Kirk, K.A. and Rutsky, E.A. (1984) Dialysis Ischemic Heart Disease. Insight from Coronary Angiography. Kidney International Journal, 25, 653-659. http://dx.doi.org/10.1038/ki.1984.70

[6] Umana, E., Ahmed, W. and Alpert, M.A. (2003) Valvular and Perivalvular Abnormalities in End-Stage Renal Disease. American Journal of Medical Science, 325, 237-242. http://dx.doi.org/10.1097/00000441-200304000-00010

[7] Mizutani, K., Naruko, T., Komatsu, R. and Ueda, M. (2011) Enhanced Expression of Neopterin in Accumulated Macrophages at Aortic Valve Stenosis in Patients with Hemodialysis. Journal of American College of Cardiology, 57, E1303. http://dx.doi.org/10.1016/S0735-1097(11)61303-X 
[8] Lentine, K.L., Costa, S.P., Weir, M.R., Robb, J.F., Fleisher, L.A., Kasiske, B.L. and Carithers, R.L. (2012) Cardiac Disease Evaluation and Management among Kidney and Liver Transplantation Candidates. A Scientific Statement from AHA/ACCF. Circulation, 126, 1-47.

[9] Bonow, R.O., Carabello, B.A. and Chatterjee, K. (2008) Focused Update Incorporated into the ACC/AHA 2006 Guidelines for the Management of Patients with Valvular Heart Disease: A Report of ACC/AHA Task Force on Practice Guidelines. Circulation, 118, e523-e661.

[10] Abbott, K.C., Hshieh, P. and Cruess, D. (2003) Hospitalized Valvular Heart Disease in Patients on Renal Transplant Waitlist: Incidence, Clinical Correlates and Outcomes. Clinical Nephrology, 59, 79-87. http://dx.doi.org/10.5414/CNP59079

[11] Sharma, A., Gilbertson, D.T. and Herzog, C.A. (2010) Survival of Kidney Transplantation Patients in the United States after Cardiac Valve Replacement. Circulation, 121, 2733-2739. http://dx.doi.org/10.1161/CIRCULATIONAHA.109.912170

[12] Shirali, A.C. and Bia, M.J. (2008) Management of Cardiovascular Disease in Renal Transplant Recipients. Clinical Journal of the American Society of Nephrology, 3, 491. http://dx.doi.org/10.2215/CJN.05081107 